\title{
Bands and bypasses: statistical limitations of regression analysis
}

\author{
Edward Livingston
}

Published online: 30 January 2009

(C) Springer Science+Business Media, LLC 2009

Lancaster and Hutter assessed the ACS NSQIP database for bariatric surgical outcomes. They concluded that "Compared with LRYGB, ORYGB is associated with higher 30-day mortality, and a higher risk-adjusted major complication rate. Although ORYGB may sometimes be indicated, a laparoscopic approach may be safer for RYGB when feasible" [1]. These conclusions are misleading.

The authors found that patients who underwent open gastric bypass operations were much more ill than those who underwent laparoscopic bypass procedures, with higher incidences of diabetes, hypertension, functional dependence, and dyspnea. They also had higher ASA scores, were older, and had higher body mass indexes. The authors' conclusion is that, despite the clearly higher risk for patients who undergo open operations, risk-adjusted analysis using regression techniques yielded a higher risk for adverse outcomes in the open bypass group. This conclusion is misleading. The authors reported a c-index for the regression of 0.633. Logistic regression, as used by the authors, models how well outcomes can be explained by patient characteristics that are thought to be predictive of those outcomes. When used, these models must be assessed in terms of their efficacy in explaining the phenomena being predicted. One measure of this efficacy is the c-index.

The c-index reports how well a model classifies the outcomes. Logistic regression models a binary phenomenon, such as alive vs. dead. The c-index measures how well the model predicts that outcome. A model that does not explain the data at all will have a 50/50 chance of arriving at an alive or dead conclusion. Thus, a model that does not explain the data at all will have a c-index of 0.5. One that always identifies alive or dead correctly every time will have a c-index of 1.0. It is generally agreed that the c-index should exceed 0.85 to assume that outcomes are appropriately modeled by a logistic-regression procedure.

The authors' c-index of 0.633 places their model as being slightly better than random at predicting whether patients who underwent bariatric operations lived or died or had complications. This means that their "risk adjustment" was not effective. It also means that there are many factors that contribute to gastric bypass complications that were not accounted for in their model. If those factors had been identified, entered into the model, and yielded a highfidelity model of gastric bypass outcomes, then, quite possibly, open and laparoscopic approaches would no longer be significant.

Regression modeling is commonly used to determine what factors contribute to clinical outcomes. It is important for readers of medical literature to understand what these models can and cannot accomplish and how results from modeling studies must be interpreted in the context of the model's efficiency in describing the phenomenon being studied.

\section{Reference}

1. Lancaster RT, Hutter MM (2008) Bands and bypasses: 30-day morbidity and mortality of bariatric surgical procedures as assessed by prospective, multi-center, risk-adjusted ACS-NSQIP data. Surg Endosc 22:2554-2563

E. Livingston $(\bowtie)$

Surgery, UT Southwestern Medical Center, Dallas, TX, USA

e-mail: edward.livingston@utsouthwestern.edu 